\section{Perfil epidemiológico das mortes \\ maternas ocorridas no Rio \\ Grande do Sul, Brasil: 2004-2007}

\section{Epidemiological profile of maternal deaths in Rio Grande do Sul, Brazil: 2004-2007}

\section{Ioná Carreno'}

\section{Ana Lúcia de Lourenzi Bonilha"}

\section{Juvenal Soares Dias da Costa"II}

' Centro de Ciências Biológicas e da Saúde; Curso de Enfermagem do Centro Universitário UNIVATES, RS.

" Programa de Pós-Graduação em Enfermagem da Universidade Federal do Rio Grande do Sul, RS.

III Programa de Pós-Graduação em Saúde Coletiva da Universidade do Vale do Rio dos Sinos UNISINOS, RS.

Correspondência: Ioná Carreno. Avenida Avelino Talini n 171, Bairro Universitário - Cidade Lajeado - Rio Grande do Sul CEP 95900-000. E-mail: icarreno@univates.br

\section{Resumo}

Foi analisado o perfil epidemiológico das mortes maternas ocorridas no período de 2004-2007, no Rio Grande do Sul, através da Razão de Mortalidade Materna e da Razão de Mortalidade Materna Específica. Foram utilizados dados do Sistema de Informações de Saúde, sendo observados 323 óbitos maternos. Para a análise dos indicadores foi empregada a regressão de Poisson e testes estatísticos. Observou-se que no Estado houve um decréscimo na razão de mortalidade materna $(0,98)$, contudo não foram encontradas diferenças nas medidas de estimativas (IC95\% 0,87-1,10). A RMME foi maior em mulheres com mais de 40 anos, com baixo nível de escolaridade, de cor preta e sem companheiro. O período de maior estimativa de risco na morte materna foi durante a gravidez e o parto, e entre as principais causas diretas observadas foram constatadas hipertensão arterial e hemorragia. A mortalidade materna é um indicador importante que deve ser enfrentado e reduzido, pois a maior parte dos óbitos pode ser evitada.

Palavras-chave: Mortalidade Materna. Saúde da mulher. Estatísticas vitais. Saúde reprodutiva. Indicadores Básicos de Saúde. 


\section{Abstract}

This study analyzed the epidemiological profile of maternal deaths that occurred from 2004 to 2007 in Rio Grande do Sul, by means of Maternal Mortality Rates and Specific Maternal Mortality Ratio. Data was obtained from the Health Information System database and 323 maternal deaths were identified. In order to analyze indicators, Poisson regression and statistical tests were carried out. A decrease in maternal mortality rates (0.98) was identified, although there was no difference in estimate measures (CI95\% 0.87-1.10). Maternal deaths were more frequent in women who were over 40 years old, had low schooling, black skin and no partners. The period of highest risk of maternal death was during pregnancy and birth, and the main direct causes were arterial hypertension and bleeding. Maternal mortality is an important issue to be confronted and reduced, given most maternal deaths could have been avoided.

Keywords: Maternal mortality. Women's health. Vital statistics. Reproductive health. Health Status Indicators.

\section{Introdução}

A saúde materna tem se destacado no cenário político internacional, integrando o quinto objetivo dos "Objetivos de Desenvolvimento do Milênio" (ODM) das Nações Unidas, referendados em 2000, sendo uma das metas a redução da taxa de mortalidade materna em 75\% de 1999 até $2015^{1,2}$.

A Organização Mundial da Saúde apresentou um comparativo entre os anos de 1990 e 2008 sobre o indicador da mortalidade materna no mundo neste período, em que foi identificado um decréscimo na razão de mortalidade materna (RMM) de $34 \%$. Em 2008, a estimativa de ocorrência foi de 358 mil óbitos maternos no mundo, sendo a RMM de 260 óbitos para cada 100 mil nascidos vivos, o que significa um risco de morte materna a cada 140 gestações. Entretanto, foi mantida a discrepância entre os países desenvolvidos e os em desenvolvimento. Nos países desenvolvidos foram encontradas as menores RMMs, com 14 óbitos para cada 100 mil nascidos vivos. Por sua vez, nos países em desenvolvimento a RMM foi de 290 óbitos para cada $100 \mathrm{mil}$ nascidos vivos, sendo que a maioria dos casos se concentra na África e na Ásia. Deve ser destacado que na África - por exemplo, em Angola - ocorre uma morte materna para cada 29 gestações, sendo este um dos mais altos valores mundiais ${ }^{2}$.

Na América Latina, aproximadamente 15 mil mulheres, morrem por ano devido a complicações na gravidez, no parto ou no puerpério ${ }^{3}$. O Chile e o Uruguai foram os países que apresentaram as melhores RMMs, sendo 26 e 27 em 100 mil nascidos vivos, respectivamente ${ }^{4}$. Em países com maiores desigualdades sociais e econômicas, como a Bolívia, a RMM é de 180 em 100 mil nascidos vivos ${ }^{2}$.

O Brasil, na mesma estimativa da OMS, em 2008 apresentou a RMM de 58 óbitos para cada 100 mil nascidos vivos, representando 1.800 óbitos e possibilidade de morte materna a cada 860 gestações ${ }^{2}$. Porém, segundo o Sistema de Informações sobre Mortalidade 
(SIM) do Ministério da Saúde (MS), no mesmo ano ocorreram 1.691 óbitos maternos ${ }^{5}$.

Percebe-se que há uma diferença entre as informações, mas, mesmo assim, qualquer que seja a fonte, o número de óbitos maternos continua sendo elevado. Sabe-se que no Brasil os óbitos maternos são subestimados ${ }^{6}$ e que as investigações sobre óbitos maternos têm sido implementadas no país nos últimos anos, o que reconhecidamente aumenta o número de mortes maternas registradas?

Ressalta-se que a razão de mortalidade materna em países em desenvolvimento permanece bastante acima do preconizado pela OMS (RMM abaixo de 20 óbitos em 100 mil nascidos vivos) ${ }^{2}$. Segundo o MS, a mortalidade associada ao ciclo gravídico-puerperal e ao aborto não aparece entre as dez primeiras causas de óbito entre as mulheres de 10 a 49 anos; no entanto, a gravidade do problema é evidenciada quando relacionado à mulher saudável no período reprodutivo, sendo estas mortes evitáveis em $92 \%$ dos $\operatorname{casos}^{8}$ se as condições de saúde locais forem semelhantes às dos países desenvolvidos ${ }^{9}$.

Desta forma, destaca-se que a mortalidade materna é um indicador importante porque reflete as condições socioeconômicas do país e a qualidade de vida de sua população, assim como das políticas públicas que promovem as ações de saúde coletiva ${ }^{2,9}$. Desta forma, as complicações maternas que levam ao óbito são quase sempre devidas à inadequada e/ou tardia assistência à saúde ${ }^{8}$.

No Brasil, as principais usuárias do Sistema Único de Saúde (SUS) são as mulheres e, destas, $65 \%$ encontram-se na faixa etária entre 10 e 49 anos $^{1}$; desta forma, o óbito materno refere-se a mulheres jovens, em idade reprodutiva e produtiva e em construção de um núcleo familiar. A evitabilidade deste óbito significa oportunizar a estruturação familiar, a construção do vínculo mãe-bebê, proporcionar o aleitamento materno; evitar o abandono, os maus tratos à criança e tantas outras situações difíceis que poderão surgir. Portanto, a evitabilidade desta morte torna-se imprescindível.
Este trabalho teve como objetivo estabelecer o perfil epidemiológico das mortes maternas ocorridas entre os anos de $2004 \mathrm{e}$ 2007 no Estado do Rio Grande do Sul, Brasil, e, a partir dele, conhecer as características sociodemográficas e reprodutivas destas mulheres, descrevendo fatores associados aos óbitos maternos.

\section{Métodos}

Entre os anos de 2004 e 2007 foi realizado um estudo ecológico descrevendo as mortes maternas no Estado do Rio Grande do Sul, Brasil. Foram utilizados dados secundários da base de dados do Sistema de Informações de Saúde disponível no Departamento de Informática do SUS - DATASUS. Deste banco de dados nacional foram utilizados o Sistema de Informações sobre Mortalidade $(\mathrm{SIM})^{5}$ e o Sistema de Informações sobre Nascidos Vivos (SINASC) ${ }^{10}$, do período de 2004 a 2007. Foram obtidos, no SIM e no SINASC, o número de mortes maternas e o total de nascidos vivos nesse período.

Foram construídos dois indicadores: a Razão de Mortalidade Materna [ $\left(\mathrm{n}^{\circ}\right.$ de óbitos maternos/no de nascidos vivos no mesmo período) x 100.000] e a Razão de Mortalidade Materna Específica (RMME) que apresenta duas fórmulas: para as variáveis sociodemográficas [(ex.: $\mathrm{n}^{\circ} \mathrm{de}$ óbitos maternos na faixa etária dos 20 aos 24 anos $/ \mathrm{n}^{\circ}$ de nascidos vivos de mães entre 20 e 24 anos, no mesmo período) x 100.000] e para as variáveis obstétricas [(ex.: $\mathrm{n}^{\circ}$ de óbitos maternos por eclâmpsia/ $\mathrm{n}^{\circ}$ de nascidos vivos, no mesmo período) x 100.000] $]^{3}$. Em vários estudos, a RMM é chamada de "taxa" ou "coeficiente", mas estes termos só poderiam ser utilizados se o denominador fosse o número de gestações, dado este de difícil obtenção. Neste estudo foi utilizada a expressão "razão", preconizada pelo Ministério da Saúde, uma vez que o seu numerador foi o número de óbitos maternos, e o denominador o número total de nascidos vivos; o resultado foi multiplicado por 100 mil seguindo o padrão internacional adotado ${ }^{3}$. 
Utilizou-se o indicador RMM para análise dos dados do Rio Grande do Sul e para as sete macrorregiões do estado: Norte, Sul, Vales, Centro-Oeste, Metropolitana, Missioneira e Serra.

A RMME foi utilizada para analisar a estimativa de possibilidades dos óbitos maternos em relação às variáveis sociodemográficas: faixa etária, raça/cor, escolaridade e estado civil; e utilizada também para as variáveis obstétricas: período de ocorrência do óbito (durante gravidez, parto ou aborto; durante o puerpério, até 42 dias; de 43 dias a 1 ano), tipo de causa obstétrica (direta, indireta e não especificada) e causa obstétrica direta segundo a Classificação Internacional de Doenças (CID) 10, Capítulo XV (Hipertensão: categoria O11, O13, O14, O15 e O16; Hemorragia: categoria O20, O44, O45, O46, O67, O71.0, O71.1e O72; Infecção Puerperal: categoria 085 e 086; Gravidez que termina em aborto: categoria $\mathrm{O} .00$ a 0.08 ; Aborto: categoria 0.03 a O. 07); esta classificação segue a orientação preconizada pelo Guia deVigilância Epidemiológica do Óbito Materno do Ministério da Saúde ${ }^{3}$.

A entrada de dados foi realizada por meio do Programa Excel. As estimativas entre as razões de mortalidade materna foram analisadas através dos intervalos de confiança de $95 \%$. O erro padrão das razões foi estabelecido a partir da fórmula (razão/ população) x $100000^{11}$.

As razões de mortalidade materna nas regiões do Estado foram analisadas por meio da regressão de Poisson ${ }^{11}$, seus respectivos intervalos de confiança de 95\% e teste estatístico (teste de Wald) no Programa Stata versão 9.0. A distribuição de Poisson se caracteriza pela contagem do número de ocorrências de um evento, que acontecem de forma independente e ao acaso, constituída por dados discretos, em indivíduos ou unidades de tempo. Desta forma, as contagens de óbitos seguem uma distribuição de Poisson ${ }^{12}$. O coeficiente de regressão mostrou a variação nas razões de mortalidade materna no período analisado.

Foi utilizado o Programa Epi-Info para cálculo do teste Qui-quadrado de tendência linear para as variáveis numéricas discretas (idade e escolaridade) e Qui-quadrado de Pearson para as variáveis categóricas (cor da pele e estado civil). Para o cálculo dos testes foram desprezadas as informações ignoradas.

Este estudo foi aprovado pelo Comitê de Ética em Pesquisa do Centro Universitário UNIVATES, sob protocolo $n^{\circ} 037 / 10$, e os autores seguiram os procedimentos éticos conforme a Portaria Ministerial 196/1996 e declaram que não há conflito de interesses.

\section{Resultados}

Entre os anos de 2004 e 2007 ocorreram 323 óbitos maternos no Rio Grande do Sul. Neste período percebeu-se um decréscimo nos valores da RMM. Em 2004, a RMM foi de $56,9(n=87)$ e em 2007 a RMM foi de 52,5 ( $n=$ 70) em 100 mil nascidos vivos; entretanto, os intervalos de confiança não mostraram diferenças entre as razões. Deve-se destacar que a RMM de 2007 foi a menor ocorrida nesse período no Rio Grande do Sul (Tabela 1).

A análise pela Regressão de Poisson confirmou essa diminuição $(0,98)$ no Estado. Entretanto, não foram significativas as diferenças entre as RMM nos anos estudados (IC95\% 0,87-1,10, Teste de Wald 0,78). Por

Tabela 1 - Razões de mortalidade materna ( por 100 mil nascidos vivos) e respectivos intervalos a 95\% de confiança. Estado do Rio Grande do Sul, Brasil, 2004-2007.

Table 1 - Maternal mortality ratios (per 100,000 live births) and 95\% confidence intervals. Rio Grande do Sul, Brazil, 2004-2007.

\begin{tabular}{lcccc}
\hline Ano & No Óbitos & $n^{\circ}$ nascidos-vivos & RMM & IC 95\% \\
\hline 2004 & 87 & 153015 & 56,9 & $45,0-68,8$ \\
2005 & 82 & 147199 & 55,7 & $43,6-67,8$ \\
2006 & 84 & 141331 & 59,4 & $46,7-72,1$ \\
2007 & 70 & 133401 & 52,5 & $40,2-64,8$ \\
\hline
\end{tabular}


outro lado, identificam-se nas sete macrorregiões do Rio Grande do Sul reduções estatisticamente significativas nas regiões Norte (IC95\% 0,78-0,98 p = 0,02), Sul (IC95\% 0,78$0,95 \mathrm{p}<0,01$ ) e nos Vales (IC95\% 0,76-0,96 $\mathrm{p}<0,01)$. Não foram observadas mudanças nas regiões Centro-Oeste (IC95\% 0,88-1,07 $\mathrm{p}<0,53$ ), Metropolitana (IC95\% 0,87-1,12 $\mathrm{p}<0,86$ ) e Missioneira (IC95\% 0,93-1,17 $\mathrm{p}<0,48)$, mas foi constatado aumento na RMM na região da Serra (IC95\% 1,52-2,11 $\mathrm{p}<0,001$ ) (Tabela 2).

Observou-se uma relação direta entre a RMM e a idade das mulheres. À medida que as faixas etárias se elevavam, maiores as possibilidades de morte materna. O teste de tendência linear apenas não foi significativo no ano de 2005 ( $p$ = 0,06). Em 2007 foram observadas oito mortes maternas na faixa etária de 40 a 49 anos, o que representou uma RMME de 132,4 em 100 mil nascidos vivos. Nesse mesmo ano, a faixa etária dos 20 aos 29 anos apresentou os menores índices, ocorreram 36 mortes maternas, e a RMME foi de 37,5 em 100 mil nascidos vivos. Não houve nenhum registro de óbito materno entre as idades de 10 a 14 anos, no período de 2004 a 2007 (Tabela 3).

Detectou-se uma relação inversa entre RMME e o nível de escolaridade. Em todos os anos analisados, o teste de Qui-quadrado para tendência linear foi significativo. Observou-se que quanto menor a escolaridade, maior a RMME. A maior RMME foi encontrada em 2007, em mulheres sem escolaridade, 256,1 em 100 mil nascidos vivos; abaixo de 7 anos de escolaridade, a RMME permaneceu elevada. Entretanto, na escolaridade superior a 8 anos a RMME reduziu drasticamente, sendo que o menor valor observado foi de 3,6 em 100 mil nascidos vivos, em 2005 (Tabela 3).

Em relação à cor/raça das mulheres que foram a óbito no período de 2004 a 2007, as mulheres de cor preta apresentaram maiores RMMEs nos anos de 2004 e 2006, com 127,0 e 213,0 em 100 mil nascidos vivos, respectivamente. Nos anos de 2005 e 2007, as maiores RMMEs foram verificadas nas mulheres classificadas com cor parda, 129,7 e 79,3 em 100 mil nascidos vivos, respectivamente. As mulheres de cor branca foram aquelas que em todo período do estudo apresentaram menores RMMEs. Em todos os anos estudados, os resultados dos testes estatísticos foram significativos, exceto em 2007 (Tabela 3).

Com referência ao estado civil, a RMME foi mais elevada entre as mulheres classificadas como solteiras em todo período do estudo, quando comparadas às viúvas/ separadas e casadas. Em todos os anos as mulheres casadas alcançaram os menores valores. Foram encontradas diferenças estatisticamente significativas nos anos de 2004 e 2005 (Tabela 3).

Quanto às variáveis sociodemográficas deve-se destacar a elevada frequência de

Tabela 2 - Coeficientes da regressão de Poisson, respectivos intervalos a 95\% de confiança e resultados de testes estatísticos relativos à razão de mortalidade materna. Estado do Rio Grande do Sul e regiões, Brasil, 2004-2007.

Table 2 - Poisson regression coefficients, 95\% confidence intervals and results of statistical tests of mortality ratios. Rio Grande do Sul, Brazil, 2004-2007.

\begin{tabular}{lccc}
\hline Variável & Regressão de Poisson & IC 95\% & Teste de Wald \\
\hline Estado RS & 0,98 & $0,87-1,10$ & 0,78 \\
Macrorregiões & & & \\
$\quad$ Centro-Oeste & 0,97 & $0,88-1,07$ & 0,53 \\
Metropolitana & 0,99 & $0,87-1,12$ & 0,86 \\
Missioneira & 1,04 & $0,93-1,17$ & 0,48 \\
Norte & 0,88 & $0,78-0,98$ & 0,02 \\
Serra & 1,79 & $1,52-2,11$ & $<0,001$ \\
Sul & 0,86 & $0,78-0,95$ & $<0,01$ \\
Vales & 0,86 & $0,76-0,96$ & 0,01 \\
\hline
\end{tabular}


Tabela 3 - Razão de Mortalidade Materna Específica (por 100.000 nascidos vivos) segundo faixa etária, escolaridade, cor da pele e estado civil. Estado do Rio Grande do Sul, Brasil, 2004-2007.

Table 3 - Specific Maternal Mortality Ratios (per 100,000 live births) according to age, education, ethnicity and marital status. Rio Grande do Sul, Brasil, 2004-2007.

\begin{tabular}{|c|c|c|c|c|}
\hline Variável & 2004 & 2005 & 2006 & 2007 \\
\hline \multicolumn{5}{|l|}{ Faixa etária } \\
\hline 10 a 14 anos & - & - & - & - \\
\hline 15 a 19 anos & 33,2 & 41,7 & 44,1 & 48,4 \\
\hline 20 a 29 anos & 46,1 & 54,7 & 51,3 & 37,5 \\
\hline 30 a 39 anos & 82,7 & 57,8 & 76,9 & 70,1 \\
\hline 40 a 49 anos & 155,8 & 139,4 & 125,8 & 132,4 \\
\hline p-valor & $<0,001^{*}$ & $0,06^{*}$ & $0,01^{*}$ & $0,01^{*}$ \\
\hline \multicolumn{5}{|l|}{ Escolaridade } \\
\hline Nenhuma & 384,1 & 167,5 & 0 & 256,1 \\
\hline 1 a 3 anos & 86,1 & 125,4 & 121,2 & 137,7 \\
\hline 4 a 7 anos & 54,2 & 44,0 & 69,6 & 33,9 \\
\hline 8 a 11 anos & 15,7 & 3,6 & 17,4 & 26,1 \\
\hline 12 anos ou mais & 24,9 & 12,1 & 8,2 & 20,8 \\
\hline $\mathrm{p}$-valor & $<0,01^{*}$ & $<0,0001^{*}$ & $<0,0001^{*}$ & $<0,001^{*}$ \\
\hline \multicolumn{5}{|l|}{ Cor/raça } \\
\hline Branca & 50,5 & 49,0 & 50,3 & 48,8 \\
\hline Preta & 127,0 & 91,7 & 213,0 & 63,5 \\
\hline Parda & 79,4 & 129,7 & 78,6 & 79,3 \\
\hline $\mathrm{p}$-valor & $0,01^{* *}$ & $<0,01^{* *}$ & $<0,0001^{* *}$ & $0,47^{* *}$ \\
\hline Amarela & - & 1098,9 & - & - \\
\hline \multicolumn{5}{|l|}{ Estado civil } \\
\hline Solteira & 70,8 & 69,5 & 67,2 & 51,8 \\
\hline Casada & 49,0 & 35,4 & 42,7 & 42,0 \\
\hline Viúva, separada, outra & 20,4 & 34,4 & 62,9 & 50,0 \\
\hline $\mathrm{p}$-valor & $0,01^{* * *}$ & $0,02^{* * *}$ & $0,20^{* * *}$ & $0,75^{* * *}$ \\
\hline
\end{tabular}

* Qui-quadrado de tendência linear (excluídas as mães com informação ignorada). ** Qui-quadrado de Pearson (excluídas as mães classificadas com cor amarela e com informação ignorada). *** Qui-quadrado de Pearson (excluídas as mães com informação ignorada).

* Chi-square for linear trend (excluding mothers with missing information). ** Chi-square test (other than mothers with skin colour classified as yellow and with missing information). ${ }^{* * *}$ Chi-square test (excluding mothers with missing information).

óbitos maternos com "informação ignorada” em relação às variáveis raça/cor, escolaridade e estado civil.

Em relação às variáveis obstétricas, observou-se o predomínio dos óbitos no período denominado "durante a gravidez, parto ou aborto" durante os anos de 2004, 2006 e 2007. No ano de 2005, prevaleceu o período "durante o puerpério, até 42 dias". Foi encontrado um óbito no período correspondente a "durante o puerpério, de 43 dias a 1 ano", em 2006, representando uma RMME de 0,7 em 100 mil nascidos vivos (Tabela 4).

Quanto ao tipo de causa obstétrica direta e indireta observou-se que, no período de 2004 a 2007, a maior ocorrência foi entre as causas obstétricas diretas seguidas das indiretas. Ao longo deste período, verificou-se diminuição das causas obstétricas diretas, sendo que em 2004 a RMME foi de 36,6 em 100 mil nascido-vivos, e em 2007 foi de 27,7 . E nesse mesmo período constatou-se aumento das causas obstétricas indiretas, sendo os valores da RMME, de 2004 e 2007, de 14,4 e 20,2, respectivamente, em 100 mil nascidos vivos (Tabela 4).

Em relação às causas obstétricas diretas, hipertensão arterial e hemorragia oscilaram entre primeiro e segundo lugares ao longo dos quatro anos de observação. Em 2004 e 2006, a principal causa foi hipertensão arterial, enquanto a hemorragia predominou nos anos de 2005 e 2007 (Tabela 4). 
Tabela 4 - Razão de Mortalidade Materna Específica (por 100.000 nascidos vivos) segundo período de ocorrência do óbito, tipo de causa obstétrica e causas obstétricas diretas. Estado do Rio Grande do Sul, Brasil, 2004-2007.

Table 4 - Specific Maternal Mortality Ratios (per 100,000 live births) according to the period of occurrence of death, type of obstetric cause and direct obstetric causes .Rio Grande do Sul, Brazil, 2004-2007.

\begin{tabular}{lcccc}
\hline Variável & 2004 & 2005 & 2006 & 2007 \\
\hline $\begin{array}{l}\text { Período de ocorrência do óbito } \\
\quad \text { Durante a gravidez, parto ou aborto }\end{array}$ & 34,0 & 18,4 & 36,1 & 27,0 \\
$\quad$ Durante o puerpério, até 42 dias & 21,6 & 25,8 & 19,1 & 24,0 \\
$\quad \begin{array}{l}\text { Durante o puerpério, de 43 dias a 1 ano } \\
\text { Causa Obstétrica }\end{array}$ & - & - & 0,7 & - \\
$\quad$ Direta & 36,6 & 34,7 & 34,0 & 27,7 \\
$\quad$ Indireta & 14,4 & 15,0 & 20,5 & 20,2 \\
Causa Obstétrica Direta & & & & \\
$\quad$ Hipertensão & 9,2 & 6,8 & 10,6 & 6,0 \\
Hemorragia & 7,2 & 8,8 & 7,1 & 8,3 \\
$\quad$ Infecção puerperal & 4,6 & 4,1 & 7,1 & 3,0 \\
$\quad$ Gravidez que termina em aborto & 8,5 & 6,8 & 2,1 & 3,0 \\
$\quad$ Aborto & 5,2 & 6,1 & 2,1 & 0,8 \\
\hline
\end{tabular}

\section{Discussão}

A Razão de Mortalidade Materna e a Razão de Mortalidade Materna Específica são potentes indicadores internacionais e nacionais preconizados pela Organização Mundial da Saúde ${ }^{2}$ e pelo Ministério da Saúde ${ }^{9}$. Eles refletem a qualidade de saúde das mulheres e da população em geral, assim como, da assistência à saúde da mulher no seu ciclo vital e, em especial, no período reprodutivo ${ }^{2,9}$.

O presente estudo investigou a mortalidade materna utilizando técnicas mais robustas. Por exemplo, em relação à RMME foram encontrados poucos estudos que analisaram este indicador, embora ele seja preconizado pelo $\mathrm{MS}^{9}$. A maioria dos estudos realizou a análise estatística descritiva, mostrando sua frequência, apresentando os resultados de forma percentual, revelando uma inversão nos resultados quando comparados à RMME.

Como limitações do estudo, pode-se referir o tempo de análise deste, o pequeno número de óbitos, a subnotificação e o sub-registro dos sistemas de informações do Brasil, além de os dados serem agregados, impedindo o controle de fatores de confundimento. Entretanto, mesmo com estas limitações, observa-se que a mortalidade materna no Rio Grande do Sul apresentou características sociodemográficas e obstétricas importantes de serem destacadas.

Cabe salientar que neste estudo foi encontrado um alto percentual de dados ignorados no SIM, nas variáveis escolaridades, cor da pele e estado civil, evidenciando limitação no Sistema de Informação. A completude destas informações pode nortear o planejamento de pesquisas, políticas públicas, intervenções e planejamento, entre outros. Autores referem que o registro dos dados no sistema de informações de saúde nacional é questionável devido ao déficit de treinamento dos profissionais ou à falta de conhecimento da notificação, gerando má qualidade e subnotificação ${ }^{13,14}$.

Em função dos óbitos serem evitáveis, a Organização Mundial da Saúde preconiza como meta a RMM de até 20 óbitos em 100 mil nascidos vivos ${ }^{2}$. O Brasil tem apresentado valores superiores ao parâmetro internacional. Em estudo sobre a mortalidade materna nas capitais brasileiras, em 2002, a RMM foi de 54,3 por 100 mil nascidos vivos, sendo que no sul do Brasil foi de 42 por 100 mil nascidos vivos 6 . O presente 
estudo mostrou que, no Rio Grande do Sul, foram observados valores menores que os do Brasil, mas no entanto ainda superiores aos preconizados pela Organização Mundial da Saúde.

No Rio Grande do Sul os coeficientes da Regressão de Poisson e seus respectivos intervalos de confiança não mostraram diferenças entre os anos estudados. Devese ressaltar que as diferenças não foram detectadas por que o período de coleta foi muito pequeno e os anos muito próximos entre si. Em estudo realizado no Paraná, no quinquênio 1998-2002, a mortalidade materna foi 2 a 3 vezes maior nos grupos de municípios mais pobres. A RMM do Paraná foi calculada em 70,6 por 100 mil nascidos vivos, destacando-se entre os principais indicadores de desigualdades sociais e em saúde ${ }^{15}$.

Nos últimos anos têm sido formuladas políticas e programas públicos voltadas à saúde da mulher, como o Programa de Humanização de Parto e Nascimento (PHPN $)^{16}$, a implantação dos comitês de mortalidade materna ${ }^{9}$ e o Pacto Nacional pela Redução da Mortalidade Materna ${ }^{17}$. Entretanto, estes parecem ter tido pouco efeito em relação aos indicadores maternos, pois as RMM ainda se mantêm elevadas, mostrando dificuldades do Brasil de atingir o objetivo 5 dos Objetivos de Desenvolvimento do Milênio (ODM) ${ }^{2}$.

No presente estudo, a maior RMM foi encontrada nas mulheres com idade de 40 à 49 anos. Estudos mostraram um risco crescente em direção às faixas etárias mais elevadas $^{18-20}$. Em estudo realizado no Rio de Janeiro, foi observado que o risco de óbito materno em mulheres acima de 40 anos de idade foi 5 vezes maior do que na faixa etária de 20 a 29 anos $^{20}$.

Observou-se que a maior RMME foi encontrada nas mulheres com menor escolaridade, em especial aquelas com menos de sete anos. Em estudo realizado no município do Rio de Janeiro, no período de 1993 a 1996, as mulheres que não tinham nem um ano de estudo apresentaram RMM de 164,1 em 100 mil nascidos vivos, e naquelas com ensino superior o RMM foi de 28,3 em 100 mil nascidos vivos ${ }^{20}$. Esses dados mostraram a estreita relação entre mortalidade materna e condições socioeconômicas. Tanto que, segundo os resultados do presente estudo, no período em questão, a partir dos oito anos de escolaridade as razões de mortalidade materna se aproximaram ou ficaram abaixo dos níveis preconizados pela Organização Mundial da Saúde.

Em relação à raça/cor, a RMME mostrou um aumento significativo em relação às mulheres pretas, seguidas das pardas. As causas de morte materna podem estar relacionadas à predisposição biológica das mulheres pretas para doenças cardiovasculares, como a hipertensão arterial ${ }^{9}$. Mas também deve-se chamar a atenção para as desigualdades sociais relacionados à dificuldade de acesso; para a baixa qualidade do atendimento recebido; e para a falta de ações e capacitação de profissionais de saúde voltadas para os riscos específicos aos quais as mulheres pretas estão expostas ${ }^{9-21}$. Corroborando estes achados, em estudo realizado pelo Ministério da Saúde, entre o período de 2000 e 2003, as mulheres de cor preta/parda apresentaram $41,5 \%$ dos óbitos maternos, seguidos das mulheres de cor branca. Esse estudo mostrou que as mulheres pretas estavam mais predispostas às doenças hipertensivas específicas da gestação (35,3\%) em relação às brancas $(22,4 \%)$; também os óbitos por aborto apresentaram maior ocorrência entre as mulheres pretas e pardas $(14,2 \% \text { e } 11 \% \text {, respectivamente })^{9}$.

Quanto ao estado civil, a RMME apresentou índices relevantes nas mulheres sozinhas (viúvas, separadas judicialmente e solteiras). Entre as mulheres viúvas foram observados valores até 62,9 em 100 mil nascidos vivos; as mulheres declaradas separadas judicialmente ou solteiras apresentaram RMMEs mais elevadas, mantendo-se entre 51,8 e 70,8 em 100 mil nascidos vivos. Entretanto, a análise não fez ajustamento por idade e o efeito pode estar confundido. Outros estudos confirmaram que o estado civil/situação conjugal mais vulnerável ao óbito materno foi não ter companheiro/ 
marido $^{6}$. A presença deste achado provavelmente pode remeter à segurança, rapidez e acesso aos serviços de saúde mais eficazes e eficientes proporcionados pela presença do companheiro, evitando complicações graves que possam levar ao óbito. Desta forma, a presença de um companheiro no período gravídico-puerperal torna-se um fator de proteção relevante na redução da morbimortalidade materna.

Em relação às variáveis obstétricas deste estudo, o período de ocorrência da maioria dos óbitos foi no momento do parto e do pós-parto imediato, apontando que estes momentos críticos devem ser adequadamente assistidos pela equipe de saúde. Estudos têm revelado que este é o momento mais vulnerável do período gravídico-puerperal, pois envolve questões administrativas e técnicas, como acesso aos serviços de saúde, qualificação profissional e tomada adequada de medidas de intervenção ${ }^{22,24}$.

Observou-se uma relação inversa entre a mortalidade materna de causas diretas e indiretas; contudo, ainda em 2007 as causas diretas predominavam. A hipertensão arterial e a hemorragia foram as principais causas de óbitos observadas. Estas causas são, em sua maioria, preveníveis com acompanhamento no pré-natal e assistência ao parto/nascimento com qualidade adequada de atendimento. Em estudo mais recente no Brasil, comparando os índices entre os anos de 1990 e 2007, entre as causas diretas foi observada uma redução de $62,8 \%$ para óbitos maternos por hipertensão; $58,4 \%$ por hemorragia; $46,8 \%$ por infecção puerperal e $79,5 \%$ por aborto; e, entre as causas indiretas, houve uma redução de $50,7 \%$ por doenças do aparelho circulatório complicadas pela gravidez, parto e puerpério ${ }^{22}$.

Em relação às causas indiretas do óbito materno, a Organização Mundial da Saúde definiu como sendo a morte resultante de doenças pré-existentes da gravidez ou aquelas doenças surgidas durante a gestação e agravadas por ela ${ }^{2}$. Em 2006, em outro estudo no RS, a AIDS foi apontada como causa do óbito materno em $8 \%$ dos $\operatorname{casos}^{18}$. As causas dos óbitos maternos vêm mudando ao longo dos anos, mas vários estudos têm observado, ainda, o predomínio das causas maternas diretas ${ }^{9,23,24}$.

No Relatório de Acompanhamento dos Objetivos de Desenvolvimento do Milênio do Ministério da Saúde, a morte materna por causa obstétrica direta apresentou uma diminuição de $56 \%$, de 1990 até 2007, sendo que a RMM foi de 55,5 por 100 mil nascidos vivos em 2007; com referência à morte materna por causa indireta, esta apresentou um aumento de $33 \%$, de 1990 a 2000 , mantendo-se estável de 2000 a 2007, sendo este provável reflexo causado pela melhoria da investigação de óbitos por causas indiretas. Desta forma, a relação entre causa direta e indireta vem apresentando uma diminuição; em 1990, a relação da causa direta era 9,4 vezes maior que a indireta; em 2007, esta relação foi de $3,2^{22}$.

A mortalidade materna, no campo da saúde e dos direitos reprodutivos, é de grande magnitude e transcendência, devendo ser percebida pelos diversos profissionais da saúde e gestores como um fato possível de ser evitado. Entretanto, os indicadores têm mostrado a dificuldade de redução e controle da morte materna no país, sendo que possivelmente não será alcançada a meta prevista pelo quinto Objetivo dos ODMs. Portanto, algumas ações e estratégias tornam-se necessárias para a diminuição da mortalidade materna. Deve ser implementado o uso de tecnologias por meio da disseminação de informações às gestantes durante o pré-natal. Desta forma, deve-se incentivar o parto normal sem intervenções desnecessárias, promover a redução da dor do parto, reservar as cesarianas para os casos de risco à saúde materna e fetal, buscar a qualificação profissional em relação à gravidez de risco e urgências obstétricas, reduzir as desigualdades na assistência, facilitar o acesso aos serviços de saúde, incentivados pelas políticas públicas.

O PHPN é uma das políticas que propõe assegurar a melhoria do acesso, da cobertura e da qualidade do acompanhamento pré-natal, da assistência às gestantes no parto e no puerpério, e ao recém-nascido, 
na perspectiva dos direitos de cidadania e isto se fundamenta na humanização da Assistência Obstétrica e Neonatal como condição primeira para o adequado acompanhamento da gestante. O programa tem como prioridades reduzir as altas taxas de morbimortalidade materna e perinatal; adotar melhorias de acesso, de cobertura e qualidade do atendimento à gestante e puérpera; e ampliar as ações de investimentos para serviços de saúde e capacitações para os profissionais ${ }^{16}$. A evitabilidade das mortes maternas é viável em nosso país, porém demanda esforços governamentais de incentivo as ações e estratégias voltadas à redução da morte materna.

\section{Referências}

1. Rosenfield A, Min CJ, Freedman LP. Making motherhood safe in developing countries. New Engl J Med 2007; 356 :1395-7.

2. World Health Organization (WHO). Trends in Maternal Mortality: 1990 to 2008. Estimates developed by WHO, UNICEF, UNFPA and The World Bank; Switzerland: World Health Organization; 2010.

3. Ministério da Saúde. Secretaria de Vigilância em Saúde. Departamento de Análise de Situação de Saúde. Guia de Vigilância Epidemiológica do Óbito Materno. Brasília: Ministério da Saúde; 2009.

4. González R. Salud materno-infantil em las Américas. Rev Chil Obstet Ginecol 2010; 75(6): 411-21.

5. Ministério da Saúde. Secretaria de Vigilância em Saúde. Banco de Dados dos Sistemas de Informação sobre Mortalidade (SIM): 1998 a 2004. Brasília: Ministério da Saúde; 2010.

6. Laurenti R, Mello-Jorge MHP, Gotlieb SLD. A mortalidade materna nas capitais brasileiras: algumas características e estimativas de um fator de ajuste. Rev Bras Epidemiol 2004; 7(4): 449-60.

7. Barros FC, Matijasevich A, Requeio JH, Giugliani E, Maranhão EG, Monteiro CA et al. Recent Trends in Maternal, Newborn, and Child Health in Brazil: Progress Toward Millennium Development Goals 4 and 5. Am J Public Health 2010; Published online ahead of print August 19: e1-e13.

8. Ministério da Saúde. Secretaria de Políticas de Saúde. Política Nacional de Atenção Integral à Saúde da Mulher. Brasília: Ministério da Saúde; 2004.

9. Ministério da Saúde. Secretaria de Políticas de Saúde. Área Técnica de Saúde da Mulher. Manual dos Comitês de Mortalidade Materna. (3 ${ }^{\mathrm{a}}$ ed.) Brasília: Ministério da Saúde; 2007.

10. Ministério da Saúde. Secretaria de Vigilância em Saúde. Banco de Dados dos Sistemas de Informação de Nascidos Vivos (SINASC): 1998 a 2004. Brasília: Ministério da Saúde; 2010.
11. Rosenberg D. Trend Analysis and Interpretation. Key Concepts and Methods for Maternal and Child Health Professionals. Maryland: Division of Science, Education and Analysis Maternal and Child Health Information Resource Center; 1997.

12. Altman DG. Practical Statistics for Medical Research. London: Chapman \& Hall; 1997.

13. Souza MH, Cecatti JG, Hardy EE, Serruya SJ. Morte materna declarada e o relacionamento de sistemas de informações em saúde. Rev Saúde Publica 2007; 41(2): 181-9.

14. Laurenti R, Buchalla CM, Lolio CA, Santo AH, Mello Jorge MH. Mortalidade de mulheres em idade fértil no município de São Paulo (Brasil), 1986: II-Mortes por causas maternas. Rev Saúde Pública 1990; 24(6): 468-72.

15. Soares VMN. Desigualdades na saúde reprodutiva das mulheres no Paraná. Rev Bras Epidemiol 2007; 10(3): 293-309.

16. Ministério da Saúde. Secretaria de Políticas de Saúde. Programa de Humanização no Pré-natal e Nascimento. Brasília: Ministério da Saúde; 2000.

17. Ministério da Saúde. Secretaria de Atenção à Saúde. Departamento de Ações Programáticas estratégicas. Pacto Nacional pela redução da mortalidade materna e neonatal. Brasília: Ministério da Saúde; 2004.

18. Riquinho DL, Correia SG. Mortalidade materna: perfil sócio-demográfico e causal. Rev Bras Enfermagem 2006; 59(3): 303-7.

19. Ramos JGL, Martins Costa S, Vettorazzi-Stuczynski J, Brietzke E. Morte materna em hospital terciário do Rio Grande do Sul - Brasil: um Estudo de 20 anos. Rev Bras Ginecol Obstet 2003; 25 (6): 431-6.

20. Theme-Filha MM, Silva RI, Noronha C. Mortalidade materna no Município do Rio de Janeiro, 1993 a 1996. Cad Saúde Pública 1999; 15(2): 397-403.

21. BRASIL. Objetivos de Desenvolvimento do Milênio Relatório Nacional de Acompanhamento. Brasília: IPEA; 2010. 
22. Laurenti R, Mello-Jorge MHP, Gotlieb SLD. Reflexões sobre a mensuração da mortalidade materna. Cad Saúde Pública 2000; 16(1): 23-30.

23. Martins AL. Mortalidade materna em mulheres negras no Brasil. Cad Saúde Pública 2006; 22(11): 2473-9.
24. Rosenstein MG., Romero M., Ramos S. Maternal mortality in Argentina: a closer look at woman who die outside of the health system. Matern Child Health J 2008; 12 (4): 519-24.

Recebido em: 16/04/2011

Versão final apresentada em: 22/09/2011

Aprovado em: 12/11/2011 